\title{
The relationship between the condition number, RGA and interaction in multivariable systems
}

\author{
Aref Shahmansoorian, Sahar Jamebozorg \\ EE Department, Imam Khomeini International University, Qazvin, Iran
}

\section{Email address:}

shahmansoorian@eng.ikiu.ac.ir (A. Shahmansoorian), sahar.jamebozorg@yahoo.com (S. Jamebozorg)

\section{To cite this article:}

Aref Shahmansoorian, Sahar Jamebozorg. The Relationship Between the Condition Number, RGA and Interaction in Multivariable Systems. Applied and Computational Mathematics. Vol. 3, No. 4, 2014, pp. 121-124. doi: 10.11648/j.acm.20140304.12

\begin{abstract}
One of the most widely used input and output controllability measure is relative gain array (RGA). RGA measures input-output interaction in multi input multi output (MIMO) systems. The other significant measure in use is the smallest singular value of frequency subordinate. The condition number is defined as the ratio between the largest and smallest singular values of a system. In this paper, the relationship of relative gain array (RGA) with condition number and interaction as well as condition number in relation to interaction will be investigated respectively. The results indicate that the parameters under investigation are not always correlated, that is, the two-way relationship is not established between them all the time.
\end{abstract}

Keywords: Relative Gain Array, Condition Number, Interaction, MIMO Systems

\section{Introduction}

Condition number and RGA are two input-output controllability measures which often are used to determine inputs and outputs. Condition number plays an important role in the numerical linear algebra and also measures the sensitivity to the changes $[1,2]$. Condition number is the ratio between the largest and smallest singular value of the system. The condition number is defined as:

$$
\begin{gathered}
\gamma(G)=\frac{\bar{\sigma}(G)}{\underline{\sigma}(G)} \\
\bar{\sigma}(G)=\max \frac{\|G u\|_{2}}{\|u\|_{2}}, \underline{\sigma}(G)=\min \frac{\|G u\|_{2}}{\|u\|_{2}}
\end{gathered}
$$

In this equation $\|u\|_{2}$ is indicative of usual Euclidian norm [3]. Condition number of a matrix is always bigger than or equal to 1 . For example, a condition number of the unit matrix equals to 1 . If a condition number of a matrix is not "too big", it is said to be Well-Conditioned matrix. A matrix with a big condition number is said to be Ill-Conditioned matrix.

For a MIMO system with $\mathrm{p}$ inputs and q outputs, when the system is operated in an open-loop condition as shown in Figure 1(a), with the assumption that a change in input $\Delta \mathrm{u}_{\mathrm{r}}$ is applied and other inputs remains constant, the output of the system will be affected consequently, and corresponding changes will be shown as $\left[\Delta \mathrm{y}_{1}, \ldots, \Delta \mathrm{y}_{\mathrm{q}}\right]^{\mathrm{T}}$. In this conditions, the steady-state gain between the rth input and the jth output (when $\Delta \mathrm{u}_{\mathrm{k}}=0, \mathrm{v} \neq \mathrm{r}$ ) amounts to $\mathrm{k}_{\mathrm{jr}}=\Delta \mathrm{y}_{\mathrm{j}} / \Delta \mathrm{u}_{\mathrm{r}}$. In the other case, as shown in Figure 1 (b) - assuming that the loop $\mathrm{u}_{\mathrm{r}}-\mathrm{y}_{\mathrm{j}}$ par is open - While all other loops are closed with a perfect controller, all output will remain unchanged. In this condition, the steady-state gain would be $\mathrm{l}_{\mathrm{jr}}=\Delta \mathrm{y}_{\mathrm{j}} / \Delta \mathrm{u}_{\mathrm{r}}$ (when $\left.\Delta y_{k}=0, \forall k \neq j\right)$. The relative gain for pair the rth input and the jth output, the ratio of open loop gain to closed loop gain is defined as follows:

$$
\begin{aligned}
& \lambda_{j r}=\frac{k_{j r}}{l_{j r}} \\
& =\frac{\left(\Delta y_{j} / \Delta u_{r}\right) \text { all loops are open }}{\left(\Delta y_{j} / \Delta u_{r}\right) \text { all loops are closed except for the } u_{r}-y_{j} \text { pair }}
\end{aligned}
$$

$\lambda_{\mathrm{jr}}$ is a parameter to measure the degree of interaction between system input $u_{r}$ and the system output $y_{j}$. And shows the ratio of the effect of input $u_{r}$ on output $y_{j}$ when all loops open to the effect when all the other loops are closed [4, $5,6]$. 


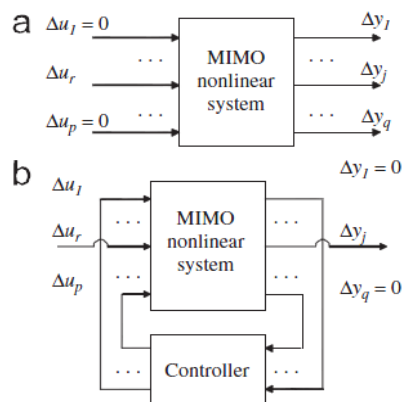

Figure 1. Relative gain array: (a) the open-loop performance (b) closed-loop performance

In 1966, Bristol [4] used the similarity between the definition of condition number and 2-norm of the RGA. However in 1985, for the first time the exact relationship between the condition number and the RGA was investigated and broached by Grosdidier et al. [5]. In 1987, the results were expanded by Morari and Skogestad [6] and also Nett and Manousiouthakis [7]. Although these results are very handy in the area of controlling, to some extent are facing constraints. In second section of this paper, the relationship between relative gain array (RGA) and condition number will be discussed. In third section, the relation between RGA and the interaction will be broached and in the fourth section the relationship between condition number and interaction with mentioning some examples will be discussed. In section five as a final section conclusion will be presented.

\section{The Relationship between the Relative Gain Array (RGA) and Condition Number}

To express the relationship between RGA and condition number first, it must be noticed that the RGA is scaling independent.

$$
\Lambda(G)=\Lambda\left(S_{1} G S_{2}\right)
$$

Here $S_{1}$ and $S_{2}$ are diagonal "scaling" matrices. Condition number as opposed to RGA is scaling dependent. Minimized condition number was presented as a solution to the scale-dependent problem $[5,7]$.

$$
\gamma^{*}(G)=\min _{S_{1}, S_{2}} \gamma\left(S_{1} G S_{2}\right)
$$

The relationship between relative gain matrix and condition number depends on minimized condition number and is verifiable with the help of this inequality [8].

$$
\gamma(G) \geq \gamma^{*}(G) \geq\|\Lambda\|_{m}-\frac{1}{\gamma^{*}(G)} \geq\|\Lambda\|_{m}-1
$$

And "m-norm" is defined as below:

$$
\|\Lambda(G)\|_{m} \triangleq 2 \max \left\{\|\Lambda(G)\|_{i 1},\|\Lambda(G)\|_{i \infty}\right\}
$$

Inequality (6) shows that: the large RGA elements always imply a large value of minimized condition number. This inequality also indicates that a system with large RGA elements is always ill-condition (big condition number) system. Since the ill-condition system, in the general case, has difficulty in controlling, then the system with large RGA elements are also experiencing controlling difficulties. Noticing this point is essential that a system could be ill-condition, even if all the elements of its RGA be small.

Example 1:

$$
\mathrm{G}=\left[\begin{array}{cc}
1 & -0.01 \\
1 & 0.01
\end{array}\right] \quad \gamma(\mathrm{G})=100, \gamma^{*}(\mathrm{G})=1,\|\Lambda\|_{m}=2 .
$$

In this system, inequality (6) is satisfied. And the matrix is ill-condition but the RGA elements are not large.

For a $2 \times 2$ matrix $[5,10]$ :

$$
\gamma^{*}(G)=\|\Lambda(G)\|_{i 1}+\sqrt{\|\Lambda(G)\|_{i 1}^{2}-1}
$$

Where the 1-norm of the RGA is defined as:

$$
\|\Lambda(G)\|_{i 1}=\max _{j} \sum_{i=1}^{m}\left|\lambda_{i j}\right|
$$

$\|\Lambda(G)\|_{\text {i1 }}$ is the induced 1-norm (maximum column sum) of the RGA-matrix of G. The value $\|\Lambda(G)\|$ sum and $\gamma^{*}(\mathrm{G})$ are always close to each other (especially when they are large).

$$
\begin{gathered}
\|\Lambda(G)\|_{\text {sum }}=\sum_{i j}\left|\lambda_{i j}\right| \\
\|\Lambda(G)\|_{\text {sum }}-\frac{1}{\gamma^{*}(G)} \leq \gamma *(G) \leq\|\Lambda(G)\|_{\text {sum }}
\end{gathered}
$$

For a real $3 \times 3$ matrix [9]:

$$
\gamma^{*}(G)+\frac{1}{\gamma^{*}(G)}=\|\Lambda(G)\|_{m}
$$

For a (complex) matrix of any size $[10,7]$ :

$$
\gamma^{*}(G)+\frac{1}{\gamma^{*}(G)} \geq\|\Lambda(G)\|_{m}
$$

\section{The Relationship between the Relative Gain Array (RGA) and Interaction in Multivariable Systems}

The most important issue in the design of multivariable control systems is interaction. Interaction disrupts the Closed-loop performance by turbulence between different loops. In decentralized control of multivariable systems, each system output is controlled by one and only one single system input. To control a certain output, such as $y_{\mathrm{i}}$, it is important to find a corresponding input, like $u_{\mathrm{i}}$, having the most effect on $\mathrm{y}_{\mathrm{i}}$ and meanwhile the control loop taking least impact from the rest of other loops. Most commonly used item in the determination of input-output pairs is relative gain array (RGA). Although there exist many methods for evaluating the 
interaction between multivariable systems, but RGA have many applications in this field yet. To solve the problem of input-output pairing, it is essential to notice some points. It is better to select pairs that their corresponding RGA elements are close to the unit, And to avoid using large or negative RGA elements for input-output pairing [10].

The property of the interaction effect of this multivariable system can be indicated by the value of RGA-element [11, 12]. Plants with large RGA-elements are always ill-conditioned. The large RGA-elements indicate strong interactions. But it does not mean that the plant with small RGA-elements is non interactive.

The RGA-number for a diagonal pairing is defined as:

$$
\text { RGA-number }=\|\Lambda(G)-I\|_{\text {sum }}
$$

The RGA can be used to measure diagonal dominance, by the RGA-number. The lower the RGA-number, the more preferred is the control structure and interactions effect in the system is less. But for $4 \times 4$ plants or larger, a small RGA number does not guarantee diagonal dominance. The RGA of a One-way interactive (triangular) plant is always the identity matrix or equivalently the RGA number is zero. Therefore the RGA or RGA-number provides a measure of two-way interaction. Example 2 shows the two-way interaction. The RGA only measures two-way interaction, whereas the performance relative gain array (PRGA) also measures one-way interaction. The PRGA is defined as below:

$$
\Gamma(s) \triangleq \bar{G}(s) G^{-1}(s)
$$

Where $\bar{G}$ (equal to $\operatorname{diag}\left\{g_{i i}\right\}$ ) is the block diagonal

System. The diagonal elements of the PRGA matrix are equal to the diagonal elements of the RGA [10].

Example 2: two-way interactive plant [13].

$$
\begin{gathered}
G(S)=\left[\begin{array}{cc}
\frac{-(400 S+2638)}{(S+116.275)(S+9.125)(S+20.015)} & \frac{(77.7 S+1211)}{(S+116.275)(S+9.125)(S+20.015)} \\
\frac{959000}{(S+116.275)(S+9.125)(S+20.015)} & \frac{(3279 S+8751)}{(S+116.275)(S+9.125)}
\end{array}\right] \\
G(0)=\left[\begin{array}{ll}
-0.1242 & 0.0570 \\
45.1589 & 8.2478
\end{array}\right], G^{-T}(0)=\left[\begin{array}{cc}
-2.2921 & 12.5496 \\
0.0158 & 0.0345
\end{array}\right], \Lambda(G)=\left[\begin{array}{ll}
0.2847 & 0.7153 \\
0.7153 & 0.2847
\end{array}\right]
\end{gathered}
$$
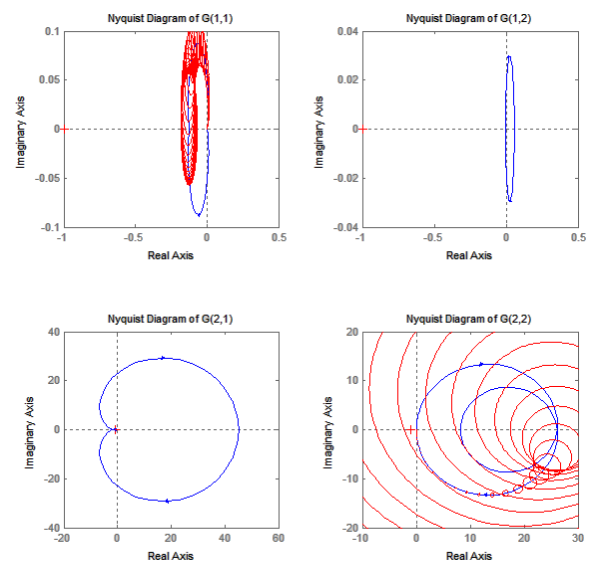

Figure 2. Gershgorin bands for open loop system

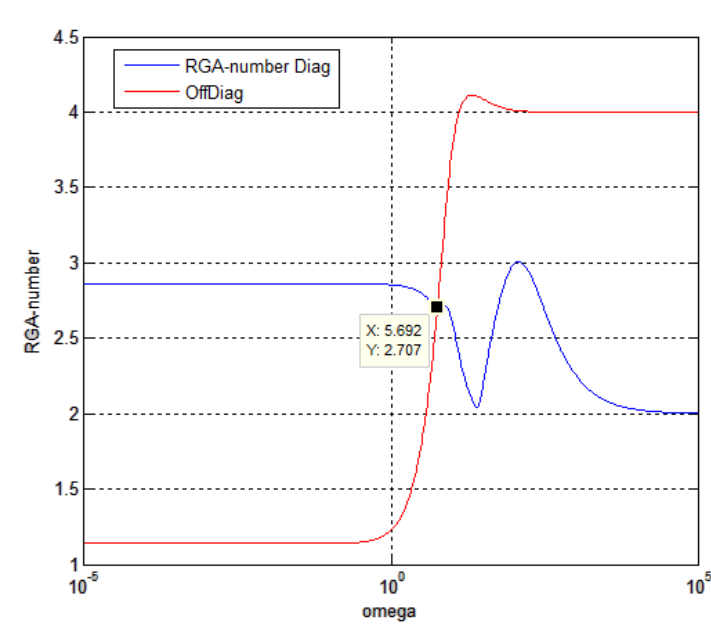

Figure 3. $R G A$ number

Gershgorin bands include origin at many frequencies and as a result, interaction of the system is high. The RGA number confirms this interaction too. The smaller RGA number, the lesser the impact of interaction in the system and consequently, the more controllable the system by means single input single output controller is. Crossing frequency - the border in which interaction direction is changed - has been marked in the figure 3 . To choose the input-output set, off diagonal elements are proper for low frequencies (because RGA number is smaller) and at high frequencies, the diagonal elements have better performance.

\section{The Relationship between the Condition Number and Interaction in Multivariable Systems}

To investigate the relationship between condition number and interaction, a few points shall be expressed first. High condition number may be made with being small the minimum single value, which is generally undesirable (or with being large the maximum single value which is not necessarily a serious problem); Or large condition number may be caused by a large minimized condition number, which is actually caused by large RGA elements, and shows fundamental problem in controlling. Large condition number indicates that the system is sensitive to uncertainty. Regarding these points, it is obvious that large condition number is not necessarily indicative of the high level of interaction in the system [14].

Example 3: ill-condition system with non interactive.

$$
G_{1}=\left[\begin{array}{cc}
100 & 0 \\
0 & 1
\end{array}\right] \gamma(\mathrm{G})=100, \gamma^{*}(\mathrm{G})=1, \Lambda(G)=I
$$

In this example condition number is large and consequently, the system will be ill-condition. However, since $G_{1}$ is diagonal, it has no interaction and relative gain matrix is unit matrix.

Example 4: ill-condition system with interaction. 


$$
G_{2}=\left[\begin{array}{cc}
1 & 10 \\
0 & 1
\end{array}\right] \gamma(\mathrm{G})=101.9902, \Lambda(G)=I
$$

$\mathrm{G}_{2}$ is a system with one-way interaction. The relative gain matrix is unit matrix (since system is triangular, RGA does not show the interaction) and the condition number of the system is large.

Two above examples show that there is not always a two-way relationship between the condition number and interaction in multivariable systems [10].

\section{Conclusion}

In this paper the relationship between the condition number, RGA and interaction in multivariate systems has been presented. We illustrated that if the condition number of system is large, the system will be ill-condition and such a system will be difficult to control. But it does not mean that the system has high interaction, because an ill-condition system does not necessarily have large RGA elements. Being high the condition number is caused by various factors, but only in the case that it is due to large RGA elements, it could be concluded that the system has high interaction. So, if RGA elements are large, condition number of system will be high (ill-condition system) and the interaction will also be high. Also if a system has high interaction, then the system has large RGA elements and will be ill-condition.

\section{Nomenclature}

$\begin{array}{ll}\text { MIMO } & \text { multi input multi output } \\ \text { RGA } & \text { Relative gain array } \\ \text { PRGA } & \text { performance Relative gain array } \\ \gamma & \text { Condition number } \\ \gamma^{*} & \text { minimized condition number } \\ \Lambda & \text { Relative gain array, with elements } \lambda_{\mathrm{jr}} \\ \sigma(G) & \text { singular values of } \mathrm{G} \\ \|X\| & \text { Euclidean norm of vector }\end{array}$

\section{References}

[1] D. Kincaid, W. Cheney, Numerical Analysis. Mathematics of Scientific Computing, Third Edition, 2008.
[2] S. Skogestad and K. Havre., The use of RGA and Condition Number as Robustness Measures,European Symposium on Computer Aided Process Engineering -Part B, vol. 20, pp. 1005-1010, 1996.

[3] D.S. Lubinsky, Condition numbers of Hankel matrices for exponential weights, Mathematical Analysis and Applications 314, 266-285, 2006.

[4] E. Bristol,On a new measure of interaction for multivariable process control, IEEE Transactions on Automatic Control, vol. 11, no. 1, pp. 133-134, 1966.

[5] P. Grosdidier, M. Morari, B.R. Holt, Closed-loop properties from steady-state gain information, Industrial \& engineering chemistry. Fundamentals - ACS, vol. 24, no. 2, pp. 221-235, 1985.

[6] S. Skogestad, M. Morari, Implications of large RGA elements on control performance, Industrial \& engineering chemistry research 26 (11), 2323-2330,1987.

[7] C. N. Nett, V.Manousiouthakis, Euclidean Condition and Block Relative Gain, Connections, Conjectures, and Clarifications, IEEE Transactions on Automatic Control, vol. 32, no. 5, pp. 405-407, 1987.

[8] K. Razzaghi, F. Shahraki, A Survey for the Selection of Control Structure for Distillation Columns Based on Steady State Controllability Indexes, Iranian Journal of Chemical Engineering Vol. 6, No. 2 (Spring), 2009.

[9] Q.Liang, Is the relative gain array a sensitivity measure?, IFAC workshop on interactions between process design and process control, London,UK,pp.133-138,1992.

[10] S. Skogestad and I. Postlethwaite, Multivariable Feedback Control: Analysis and Design (2nd Edition). UK: Wiley, 2005.

[11] A. Khaki-Sedigh, B. Moaveni, Control Configuration Selection for Multivariable Plants, LNCIS 391, Springer Verlag, 2009.

[12] B. Halvarsson, Interaction Analysis in Multivariable Control Systems Applications to Bioreactors for Nitrogen Removal, Phd Thesis, Uppsala University, Sweden,2010.

[13] M.Mousavi, M.Haeri, Welding current and arc voltage control in a GMAW process using ARMarkov based MPC, Control Engineering Practice, Vol. 19, PP.1408-1422, 2011.

[14] J.M.Maciejowski, Multivariable Feedback Design, Addison-Wesley, 1989. 